\title{
L'insuffisance des signaux de localisation pour la coordination entre la production et le transport d'électricité dans les systèmes électriques libéralisés
}

\author{
Vincent Rious ${ }^{1}$, Jean-Michel Glachant ${ }^{2}$, Yannick Perez ${ }^{3}$, Philippe Dessante ${ }^{1}$
}

\section{Résumé}

Cet article analyse comment s'organise la coordination à long terme entre la production et le transport dans un système électrique libéralisé. Nous nous appuyons sur un cadre d'analyse modulaire afin de séparer les mécanismes de coordination entre la production et le transport d'électricité en modules distincts. La structure de gouvernance du réseau de transport complète ce cadre d'analyse. Nous montrons alors que, dans une logique de complémentarité institutionnelle, cette structure de gouvernance influence les options de gestion des flux effectivement mises en œuvre par les Gestionnaires de Réseaux Transport d'électricité. Bien que les signaux de localisation soient nécessaires afin de guider l'installation de nouveaux moyens de production, les contraintes de localisation des moyens de production et la structure de gouvernance du réseau expliquent alors que ces signaux sont insuffisants pour coordonner à long terme production et transport.

\begin{abstract}
This paper analyses how the long run coordination between generation and transmission is organized in a liberalized power system. We rely on a modular analysis framework in order to split in disctinct modules the coordination mechanisms between generation and transmission of electricity. The governance structure of the power transmission grid completes this analysis framework. Then, we show that, in a logic of institutionnal complementarity, this governance structure influences the power flow management schemes that are effectively implemented by the power Transmission and System Operators. Although the locational signals are necessary to guide the location of new power plants, the locational constraints of generators and the governance structure of the network then explain that these signals are insufficient to coordinate generation and transmission to the long term.
\end{abstract}

Code JEL : D23, D62, L5, L29, L94

\section{INTRODUCTION}

Afin d'assurer un accès non-discriminatoire au réseau de transport dans les systèmes électriques libéralisés, il a été nécessaire de séparer verticalement la production en concurrence et le réseau de transport en monopole naturel. La séparation d'activités auparavant intégrées dans un monopole vertical et horizontal fait apparaître des problèmes de coordination entre les investissements en production et en réseau. Nous montrons que les

\footnotetext{
${ }^{1}$ Professeurs assistant à Supélec, Département d'Électrotechnique et de Systèmes d'Énergie, 3 rue Joliot-Curie, 91192 Gif-sur-Yvette CEDEX

2 Directeur de la «Florence School of Regulation» et de la chaire «Loyola de Palacio » à 1'European University Institute.

${ }^{3}$ Maître de conférences à l'université Paris-Sud 11, ADIS-Groupe Réseaux Jean Monnet et chercheur associé à la Chaire «Loyola de Palacio » de l'European University Institute.
} 
signaux de localisation sur le réseau de transport sont insuffisants pour réaliser cette nouvelle coordination à long terme dans un système électrique libéralisé.

Face à cette situation, Lévêque (2003) a montré qu'une autorité centralisée devait émettre des signaux de localisation à destination des producteurs pour remplacer la coordination traditionnelle. Cette autorité centralisée s'appelle le Gestionnaire du Réseau de Transport (GRT). On s'attendrait donc à ce que les GRT mettent en place les meilleurs signaux existants pour coordonner la production et le transport. Or empiriquement, on constate qu'aucun GRT ne met en œuvre concrètement les meilleurs signaux économiques disponibles. Pire, l'étude des GRT montre que ceux qui mettent en œuvre les signaux de localisation les plus incitatifs ne sont pas ceux aboutissant à la meilleure coordination avec la production. Comment expliquer ce paradoxe?

Pour répondre à cette question, nous proposons dans la première section la construction d'un GRT idéal à partir d'une analyse modulaire à la Baldwin et Clark (2000) et Wilson (2002). Ce GRT idéal nous servira de point de comparaison pour l'étude de la coordination entre production et le transport de GRT réels. Dans la seconde section, en nous appuyant sur le concept de structure de gouvernance de l'économie néoinstitutionnelle et sur les travaux d'Aoki (2001) sur la complémentarité institutionnelle, nous montrons que la structure incitative du GRT idéal est difficile à atteindre car le mode de gestion des actifs de transport d'électricité influence la mise en œuvre de la gestion des flux. Enfin, dans la troisième section, nous appliquons ce cadre d'analyse modulaire et institutionnel pour comparer deux GRT Pennsylvania, Maryland \& New Jersey (PJM), et National Grid. Nous verrons alors que les signaux de localisation ne sont pas centraux pour coordonner la production et le transport d'électricité dans les organisations réelles.

\section{1- ORGANISATION IDEALE D'UN GRT}

La gestion des flux électriques par les Gestionnaires des Réseaux de Transport comporte trois missions principales qui s'étendent du très court terme (quelques minutes à quelques heures) au très long terme (5 ans à 20 ans) (Brunekreeft et al., 2005). Ces trois missions sont : i) la gestion des externalités des flux électriques sur les réseaux; ii) le développement des capacités de transport, et enfin iii) la coordination avec les réseaux voisins. Pour étudier les multiples variantes organisationnelles existantes, nous avons montré dans Rious et al. (2008) la pertinence du cadre d'analyse modulaire à la Baldwin et Clark (2000) et Wilson (2002) qui permet de classer la diversité empirique existante. Ici nous n'avons besoin que de nous intéresser à deux missions : la gestion des externalités et le développement du réseau de transport. En choisissant pour chacune de ces deux missions la variante optimale, un GRT idéal peut être défini par la combinaison du «pricing nodal » et, dans le cadre d'une régulation bienveillante, de la minimisation du coût total du réseau (c'està-dire minimiser la somme du coût de congestion du réseau et du coût d'investissement). Nous allons détailler ces deux points et voir pour quelles raisons le pricing nodal et le développement à long terme du réseau de transport constituent les briques élémentaires de l'organisation optimale d'un GRT idéal.

Premièrement, l'optimum de gestion des externalités de réseau est obtenu à court terme par le système du pricing nodal de l'énergie. Schweppe et al. (1988) démontre qu'un appel efficace des centrales peut être obtenu grâce à un système de tarification nodale de l'énergie (aussi appelé «pricing nodal» par anglicisme) dont l'équilibrage est contraint par les externalités liées aux congestions des lignes du réseau. Cette méthode présente des vertus 
incitatives supérieures aux deux autres techniques les plus connues, le pricing zonal ${ }^{4}$ et le redispatching ${ }^{5}$. Ainsi la tarification nodale de l'énergie détermine un prix de l'énergie pour chaque nœud du réseau. Ces prix nodaux indiquent où il est préférable de produire ou de consommer un mégawatt supplémentaire compte tenu des contraintes de capacité du réseau.

La figure 1 illustre le pricing nodal sur un réseau congestionné simple à deux nœuds $\mathrm{S}$ et $\mathrm{D}$. Au nœud S, il n'y a que de la production dont le coût est faible. Au nœud D, il y a une quantité $\mathrm{Q}$ de consommation inélastique et de la production dont le coût est élevé. Ces deux nœuds sont reliés par une seule ligne électrique SD de capacité de transport K. Si on ignore la capacité limitée de transport à l'équilibre, toute la consommation est alimentée par la production du nœud $\mathrm{S}$. Le prix d'équilibre est alors unique et vaut $\mathrm{P}_{\mathrm{E}}$. Cet équilibre induit un flux sur la ligne SD qui dépasse la capacité de transport disponible $\mathrm{K}$, où $\mathrm{K}<\mathrm{Q}$. Cet équilibre n'est donc pas techniquement faisable. Pour inciter la production au nœud S et au nœud D à prendre en compte la congestion sur la ligne SD, le pricing nodal paie au producteur au nœud $S$ un prix $P_{S}$ inférieur au prix d'équilibre sans congestion $P_{E}$ et paie au producteur au nœud $D$ un prix $\mathrm{P}_{\mathrm{D}}$ supérieur à $\mathrm{P}_{\mathrm{E}}$. Dans ce cas de figure, le différentiel de valeur entre les prix nodaux reflète alors la valeur sociale des externalités de réseau. Ce différentiel génère ainsi un surplus pour le GRT appelé «rente de congestion », représentée par le rectangle gris clair sur la figure 1. Et les contraintes de capacité du réseau limitent la maximisation du surplus social d'une quantité appelée «coût de congestion », représentée par le trapèze gris foncé sur la figure 1 .

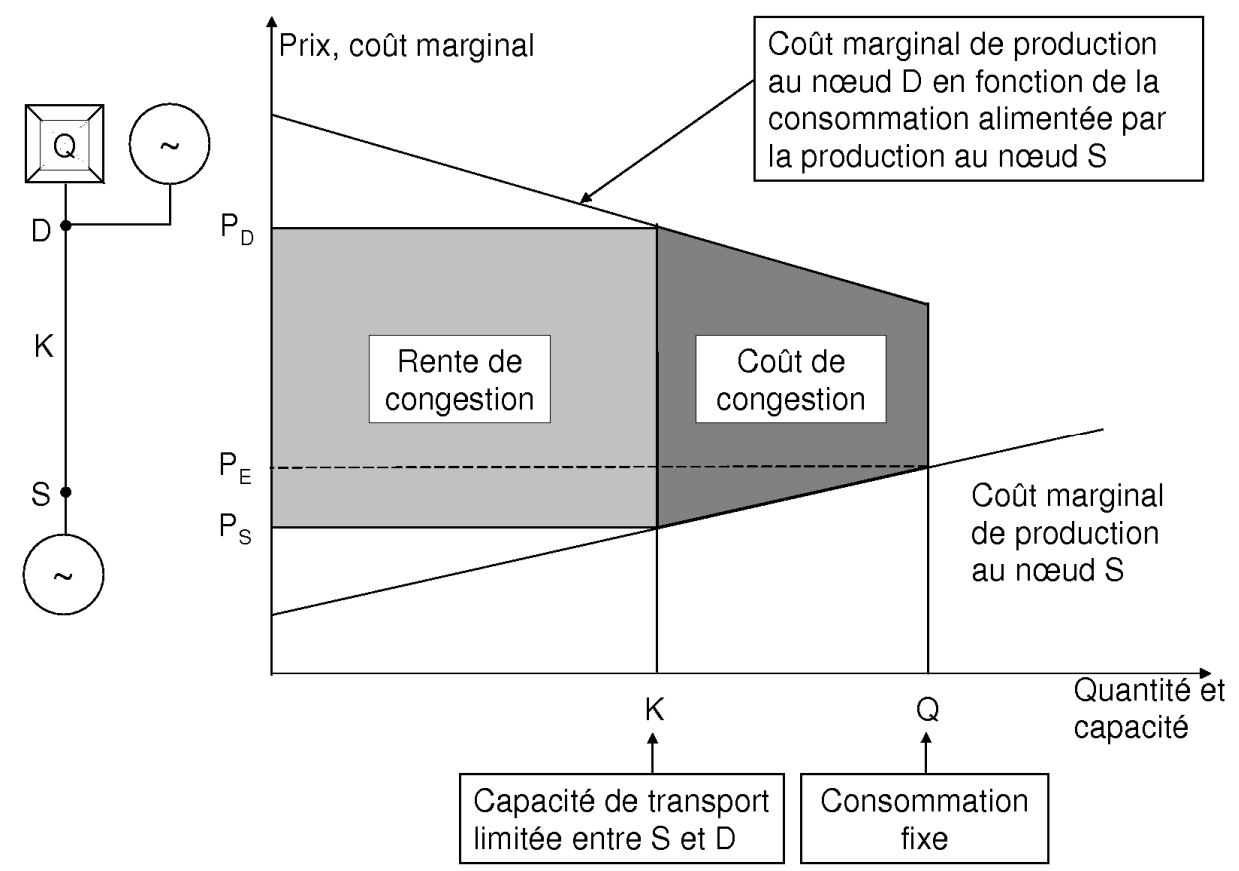

Figure 1 Représentation graphique du pricing nodal sur un réseau congestionné à deux nœuds

\footnotetext{
${ }^{4}$ Le pricing zonal est similaire au pricing nodal mais seules les contraintes de réseau les plus importantes sont internalisées dans le système de prix du marché électrique. Les autres contraintes mineures sont gérées par redispatching. Cette simplification de milliers de nœuds à quelques zones est très sensible aux erreurs et aux manipulations.

5 Lorsque les congestions sont gérées par redispatching, elles ne sont pas internalisées dans le marché électriques. Le GRT les gère après la clôture du marché en modulant la production des centrales adéquates. Le redispatching n'émet donc aucun signal de localisation, sauf pour les centrales modulées.
} 
Le second mécanisme de coordination entre production et transport est le développement à long terme du réseau de transport d'électricité. En principe, la gestion à court terme et à long terme des externalités des flux électriques peut donner des informations au GRT et aux utilisateurs du réseau sur les contraintes liées à l'état actuel de fonctionnement du réseau. Mais le GRT doit aussi chercher, à long terme, à réaliser des investissements de réseau efficaces pour lever toutes les contraintes de réseau économiquement excessives. Si l'on considère par simplification que le GRT est bénévole et efficace, il doit investir afin de réduire le coût social suscité par les externalités de réseau et pour, de façon équivalente, maximiser le surplus social.

Face à cette définition du GRT idéal, l'observation des modes d'organisations des GRT réels reflète une grande variété des options mises en œuvre et de leurs performances pour la gestion des flux électriques. Cette variété s'explique au-delà des contraintes techniques par les différentes caractéristiques des structures de gouvernance du réseau de transport.

\section{2- DES COMPLEMENTARITES ENTRE LA STRUCTURE DE GOUVERNANCE DU RESEAU ET LA GESTION DES FLUX ELECTRIQUES}

L'économie néoinstitutionnelle a montré que le mode de gestion des actifs de réseau, qu'elle appelle structure de gouvernance, est aussi important que la régulation pour une gestion efficace de ces infrastructures (Saussier et Yvrande-Billon, 2007). Dans le système électrique libéralisé, le GRT peut prendre différentes formes organisationnelles (structures de gouvernance), selon qu'il détient les droits de propriété du réseau qu'il gère, et selon la forme de régulation qui est appliquée à ce monopole 6 . La structure de gouvernance du réseau modifie les incitations que le GRT peut trouver dans sa gestion des flux électriques. Dans cette section, nous définissons tout d'abord la structure de gouvernance du réseau de transport en présentant ses principales composantes. Ensuite, nous montrons que la structure de gouvernance du réseau influence les options que le GRT met en œuvre pour gérer les flux électriques.

2- a) La gouvernance et la régulation incitative du réseau

La forme prise par la séparation du réseau de transport d'électricité constitue la base de sa gouvernance car elle détermine le degré d'incitation qui peut être introduit dans la régulation de ce monopole. La séparation du réseau de transport vis-à-vis des métiers de la production et de la commercialisation est fréquemment admise comme indispensable. Cette séparation verticale est de deux degrés d'intensité. Le premier niveau comprend toujours la séparation de la gestion du système pour les flux à court terme. En effet, le retrait de la gestion du système aux opérateurs historiques est assez facile à imposer dans un processus de dérégulation car cette activité représente un volume d'investissement et d'emploi relativement faibles. A contrario, le second degré de séparation de la propriété du réseau de transport dépend de la possibilité d'imposer aux opérateurs historiques de céder la propriété de leurs

\footnotetext{
${ }^{6}$ Rious et al. 2008 montrent aussi que les options mises en œuvre dépendent aussi de leur acceptabilité par les acteurs dominants du marché (Political Economy, voir Perez, 2002). Mais ce point n'est pas crucial pour notre argumentation.
} 
actifs de réseau. Ce niveau de séparation verticale est plus difficile à produire lors des réformes concurrentielles. En effet, le réseau, étant régulé, est une source de revenu garanti et récurrent. De ce fait, dans le contexte d'incertitude des marchés électriques où les entreprises sont également jugées sur leurs performances financières, la sécurité du revenu régulé des infrastructures de transport est attractive pour un opérateur soumis aux incertitudes du marché et aux pressions concurrentielles sur la production et la commercialisation du bien électrique. De plus, la propriété du réseau peut permettre aux opérateurs de protéger stratégiquement leurs parcs de production dans les plans de développement du réseau.

Les choix du niveau de séparation du réseau peuvent aussi être influencés par les interactions aussi appelées «effets de frontière » entre réseaux interconnectés. Si la propriété et la gestion d'un réseau à l'échelle continentale sont fortement fragmentées entre de multiples GRT distincts, il y a de nombreux «effets de frontières » entre GRT qui sont difficiles à traiter et qui peuvent atteindre des valeurs critiques pour la sécurité du système. Pour internaliser les « effets de frontières » et résoudre les problèmes associés, un remède est alors d'enlever la partie «Opération du Système » aux différents producteurs-transporteurs historiques et de l'intégrer horizontalement sur une zone géographique plus vaste englobant plusieurs zones électrique (PJM, 2004).

On peut maintenant distinguer deux grandes familles de GRT en terme de degré de séparation des activités de gestion des flux électriques et de leur propriété. Nous définissons ainsi deux types de GRT : le GRT «lourd » et le GRT « light ». Un « GRT lourd » possède et gère les infrastructures de réseau dont il est l'opérateur du système. Un «GRT light» ne possède pas les infrastructures dont il est l'opérateur du système. Ces modalités de séparation du réseau de transport sont donc importantes à la fois pour comprendre l'efficacité de la gouvernance du transport de l'électricité mais aussi sa régulation en tant que monopole. Dans le cas d'un GRT «lourd », le régulateur peut imposer une régulation incitative sur les coûts contrôlables du GRT afin de fixer le revenu de ce monopole. En effet, les risques financiers potentiels d'une régulation incitative sont acceptables pour un «GRT lourd » car il perçoit un revenu sûr et important pour l'accès à son réseau. Au contraire, il est difficile pour un régulateur d'inciter fortement un «GRT light » à cause de sa faible surface financière. C'est pourquoi les «GRT light» sont le plus souvent des entités à but non lucratif, partiellement autorégulées par les acteurs du marché de l'électricité couverts par leur zone de responsabilité (Barker et al., 1997) ${ }^{7}$.

2- b) la gouvernance du réseau et la gestion des flux sont complémentaires

Un GRT n'a pas les mêmes incitations à gérer son réseau et à y investir selon la combinaison qui existe entre sa structure de gouvernance et ses méthodes de gestion à court terme des externalités. Suivant la méthode choisie, la gestion de ces externalités peut générer une rente ou un coût pour le GRT. Ainsi le pricing nodal conduit efficacement le dispatching des utilisateurs du réseau et, en partie, leur localisation. Mais la rente de congestion y donne un signal contre-incitatif au GRT pour ces propres décisions d'investissement. En effet, le pricing nodal peut inciter un GRT maximisateur de profit à faire durer les congestions (PérezArriaga et al., 1995). On en déduit qu'un GRT internalisant les externalités des flux électriques grâce au pricing nodal devrait faire l'objet d'une régulation plus exigeante afin de

\footnotetext{
${ }^{7}$ On notera que le bon fonctionnement de ce principe d'autorégulation est efficace sous l'hypothèse toutefois qu'il n'y a pas de risque de collusion ou de capture du GRT light par un seul groupe d'intérêt (Boyce et Hallis, 2005).
} 
s'assurer qu'il fait bien coïncider la maximisation de son profit de GRT avec la maximisation du surplus social.

$\mathrm{Au}$ contraire, la méthode dite de «redispatching» est réputée inefficace pour internaliser les externalités des flux électriques car le GRT y gère les congestions hors du marché électrique. Aucun signal de localisation n'est donc transmis aux utilisateurs du réseau qui ne peuvent alors prendre de bonnes décisions de localisation. Mais cette méthode présente l'avantage de faire supporter directement par le GRT le coût des congestions découlant de l'opération du système. En conséquence, ce GRT peut maximiser son profit en comparant le coût social de court terme des congestions avec le coût à long terme d'investissement et de maintenance du réseau et maximise ainsi naturellement le surplus social. Dans ce cas, le régulateur a lui-même facilement accès au coût de congestion par l'intermédiaire du GRT.

La structure de gouvernance du réseau et la conception des modules de gestion des flux électriques doivent donc être vues comme un système global. La perfection théorique serait de combiner les options du GRT idéal avec un périmètre de GRT lourd, séparé de la production et doté d'un régulateur fort mais également incitatif. Cependant les complémentarités entre la structure de gouvernance et les modules de gestion des flux électriques conduisent à des choix sous-optimaux dans la conception de ces modules. Des arbitrages doivent alors être réalisés entre le contrôle des coûts du réseau et la conception des modules de gestion des flux électriques.

L'étude de deux GRT montrera dans la section suivante que leur structure de gouvernance influence le choix du mode de coordination entre la production et le réseau. Ainsi certains GRT favorisent l'internalisation des externalités par des prix de rareté à capacité de réseau donnée, tandis que d'autres agissent directement sur la cause des externalités en augmentant la capacité de transport via l'investissement.

\section{3- LA COMPARAISON DE DEUX GRT DE REFERENCES : PJM ET NATIONAL GRID}

L'objectif de cette partie est double. Premièrement, nous étudions les conséquences des complémentarités sur des GRT de référence pour expliquer leurs choix pour gérer les flux électriques. Nous procéderons en deux étapes. Tout d'abord, nous comparerons les options de gestion des flux de chaque GRT à celles du GRT idéal. Ensuite, nous montrerons comment la structure de gouvernance du réseau contraint la mise en œuvre de ces options. Nous montrons enfin que la structure de gouvernance peut corriger les défauts de certaines options sousoptimales de coordination.

À partir de cette comparaison, le second objectif de cette section est de montrer que les effets de complémentarités apportées par la structure de gouvernance du réseau se concentrent sur le choix entre «internaliser les externalités d'usage du réseau liées aux congestions » ou «augmenter la capacité du réseau pour traiter la cause des congestions ». Nous montrerons alors qu'il semble très difficile de réaliser conjointement ces deux tâches.

\section{3- a) Comparaison des GRT réels au GRT idéal}

Notre construction de GRT idéal va maintenant nous servir de point de comparaison pour l'étude de GRT réels. Nous considérons deux GRT de référence dans les expériences internationales de libéralisation des systèmes électriques. 
Le premier, PJM, est un GRT light structuré comme une organisation à but non lucratif qui opère dans le nord-est des États-Unis. PJM est reconnu comme un modèle car le pricing nodal lui a permis d'étendre sa zone de responsabilité sur une part conséquente du nord-est des Etats-Unis et de devenir ainsi le GRT gérant la plus importante consommation de pointe au monde (Joskow, 2006). Malgré la mise en œuvre de la meilleure méthode d'internalisation, le coût des congestions de cette zone a fortement augmenté jusqu'en 2006. En fait, avant avril 2004, aucune opportunité économique de réduction des congestions n'était prise en considération dans la planification des investissements du réseau de PJM. Les investissements du réseau n'étaient faits que pour des raisons techniques de fiabilité.

Le second modèle est le GRT anglo-gallois, National Grid. C'est un GRT lourd, propriétaire du réseau, séparé de la production, qui détient, maintient, planifie et gère le réseau anglo-gallois à court, moyen et long terme. National Grid est une entreprise privée, cotée en bourse, mais régulée par le régulateur britannique de l'énergie, l'OFGEM ${ }^{8}$ (Joskow, 2006). Ce GRT est souvent cité comme exemple à suivre pour l'efficacité de la gestion du réseau dans le cadre d'un système électrique libéralisé. Alors même que National Grid conduit son système à l'aide du redispatching et que cette option de gestion du système n'internalise pas les externalités d'usage du réseau. Mais les modalités pratiques de régulation appliquées à ce monopoleur pour la gestion de son système et le développement du réseau le poussent à investir de façon à minimiser le coût de congestion. Ses coûts de congestion et d'investissement ont ainsi été considérablement réduits.

Nous constatons que ces deux GRT n'appliquent pas la combinaison de mécanismes de coordination du GRT idéal. Et ce, bien qu'ils soient tous deux reconnus à l'échelle internationale pour la gestion de leur réseau. Par ailleurs, l'évolution du coût de congestions des GRT ne rend pas compte des signaux de localisation utilisés par chacun de ces GRT. En effet, il est paradoxal que PJM voit ses congestions s'accroître alors que ces signaux de localisation sont très incitatifs. De même, il est paradoxal que National Grid ait un bon contrôle de son coût de congestion, alors que ses signaux de localisation sont théoriquement plus faibles.

Le tableau suivant permet de synthétiser la comparaison de ces deux GRT, PJM et National Grid au GRT idéal, ainsi que l'évolution de leur coût de congestion.

\begin{tabular}{|c|c|c|c|}
\hline \multicolumn{4}{|c|}{ Tableau de comparaison des deux GRT } \\
\hline & GRT idéal & PJM & NGC \\
\hline $\begin{array}{l}\text { Gestion des } \\
\text { congestions }\end{array}$ & $\begin{array}{c}+ \\
\text { Pricing nodal } \\
\end{array}$ & $\begin{array}{c}+ \\
\text { Pricing nodal } \\
\end{array}$ & $\begin{array}{c}- \\
\text { Redispatching }\end{array}$ \\
\hline Investissement & $\begin{array}{c}\text { + Maximisation du } \\
\text { surplus social }\end{array}$ & $\begin{array}{c}\text { - Réduction des } \\
\text { congestions pas prise } \\
\text { en compte }\end{array}$ & $\begin{array}{l}+ \text { Arbitrage entre } \\
\text { coût de congestion et } \\
\text { coût d'investissement }\end{array}$ \\
\hline $\begin{array}{c}\text { Evolution des } \\
\text { Congestions }\end{array}$ & & $\eta$ & $\mathbf{y}$ \\
\hline
\end{tabular}

Sources : Joskow (2006)

3- b) Gouvernance du réseau et conséquences sur les options de gestion des congestions

La structure de gouvernance du réseau qui complète notre cadre initial d'analyse permet d'expliquer les options mises en œuvre par les GRT ainsi que leurs performances pour coordonner la production et le transport d'électricité. En particulier, le mode de séparation du

${ }^{8}$ Office of Gas and Electricity Markets 
réseau est central car il détermine la régulation la plus appropriée qui peut être appliquée au GRT.

Dans le cas de PJM, le réseau reste la propriété des opérateurs historiques. La structure de «GRT light » de PJM présente l'avantage de pouvoir s'étendre facilement pour y intégrer de nouvelles zones de marché. Cette extension est d'autant plus efficace qu'elle s'effectue grâce à la tarification nodale de l'énergie. L'usage de cette méthode s'avère obligatoire dans le cas d'un GRT light comme PJM car, par nature, PJM est insensible au coût ou à la rente de congestion issue des signaux de court terme. Néanmoins, le pricing nodal n'a pas empêché une recrudescence des congestions sur la zone de PJM.

L'accroissement des congestions a révélé en fait une coordination insuffisante entre la production et le transport au sein de PJM. Ce problème a fini par attirer l'attention du régulateur fédéral de l'énergie, la FERC (PJM, 2004). Ainsi, ce n'est que sous la pression de la FERC que PJM a défini le concept «d'infrastructures de transport planifiées pour raison économique » (Economic Planned Transmission Facilities) (Joskow, 2006). Sans la contrainte du régulateur, PJM n'aurait probablement pas pris cette initiative car son statut de GRT light ne l'y incite pas. En effet, le cœur de son activité est la gestion du réseau à court terme et c'est donc l'extension de cette activité de gestion qui guide le développement de PJM.

A l'inverse, quand le GRT possède le réseau comme National Grid, le développement de l'infrastructure est son cœur de métier. La propriété du réseau permet alors de lui imposer une régulation incitative ou des gains de productivité. Alors, lui faire supporter le coût de congestion du redispatching même partiellement l'incite à développer efficacement le réseau. En effet, grâce à la structure de gouvernance de GRT lourd de National Grid, une régulation incitative permet de maîtriser les coûts de conduite du système liés aux congestions et, dans une moindre mesure, les coûts d'investissements du réseau. Cette régulation incitative permet de compenser les défauts théoriques du redispatching liés à la non-internalisation des externalités. La régulation de la conduite et de la propriété du réseau propose à National Grid un arbitrage entre les coûts de conduite du système à court et moyen terme et les coûts d'investissements du réseau (Joskow, 2006). Dans ce cadre, la régulation incitative de la conduite du système incite National Grid à arbitrer entre les coûts de congestions et les investissements de petites tailles (avec de courtes périodes de retour sur investissement). Tandis que la contrainte budgétaire de long terme incite National Grid à arbitrer entre les investissements de petite taille et les investissements de plus grande taille, ces derniers pouvant être moins coûteux grâce aux économies d'échelle caractéristiques des réseaux électriques.

3- c) Gouvernance du réseau et coordination entre production et transport

Notre analyse permet de montrer que, pour traiter les congestions, les GRT light et les GRT lourds optent pour des méthodes fondamentalement différentes. Les GRT light comme PJM se concentrent sur l'internalisation des congestions dans un système de prix, tandis que les GRT lourds comme NGC se concentrent sur le développement du réseau.

La structure de gouvernance du réseau influence la coordination entre la production et le transport. Suivant sa forme, la gouvernance incite le GRT à se concentrer soit sur l'internalisation des congestions (à capacité de transport donnée) soit sur le développement du réseau (afin de traiter la cause de ces congestions). En pratique, il est difficile de concilier efficacement et simultanément ces deux approches de traitement des externalités.

$\mathrm{Au}$ vu des performances de PJM et National Grid, on constate que le module d'investissement du réseau tient une place centrale dans la coordination entre la production et 
le transport. Ainsi, même si le système de PJM est le mieux pourvu en signaux de localisation, l'évolution de la rente de congestion sur sa zone montre que les investissements en réseau y étaient insuffisants et que la coordination entre les investissements en production et en réseau n'y était pas satisfaisante. À l'inverse, National Grid est davantage concerné par la coordination avec la production sur le long terme. Ce qui se comprend bien puisqu'il possède le réseau et préfère le développer pour éliminer des congestions. ${ }^{9}$

Mais au regard de l'évolution des coûts de congestion, la stratégie consistant à mettre en place des signaux de localisation sur le réseau électrique est moins efficace. Les signaux de localisation sont certes nécessaires pour inciter les producteurs à prendre en compte les coûts du réseau électrique dans leur décision locale d'implantation. Mais les producteurs ont des contraintes de localisation fortes pour installer leurs nouvelles centrales. La source d'énergie primaire doit être facilement accessible. Pour les moyens de production thermique (à gaz, à charbon ou nucléaire), un cours d'eau avec un débit important est aussi nécessaire. Enfin, les producteurs doivent trouver les terrains qui satisfont ces critères à un prix raisonnable. Ces contraintes pour les producteurs conduisent à des congestions durables pour lesquelles la seule solution est de développer le réseau.

Les signaux de localisation ne sont donc que secondaires dans la coordination à long terme entre production et transport d'électricité. Et c'est l'investissement en réseau qui tient donc un rôle central dans la coordination à long terme entre production et transport, et ce pour deux raisons. Premièrement, la structure de gouvernance du réseau peut conduire à mettre en place peu ou pas de signaux de coordination. Ensuite, les signaux de coordination ne garantissent pas que la cause de la congestion, liée au manque de capacité du réseau, soit traitée.

\section{CONCLUSION}

Nous avons montré que les complémentarités entre la structure de gouvernance du réseau et les modules de gestion des flux influencent la coordination entre la production et le transport d'électricité, et ce de deux manières. Tout d'abord, la structure de gouvernance du réseau définit le degré de séparation du GRT par rapport au reste du système électrique, ce qui modifie les incitations qu'il peut trouver dans les différentes options de gestion des flux. Ainsi, selon la structure de gouvernance, seules des options sous-optimales de gestion pourront être appliquées, notamment quand les options optimales sont contre-incitatives pour le GRT dans la configuration de sa gouvernance. Mais la structure de gouvernance peut aussi corriger certains défauts de ces méthodes sous-optimales.

Ensuite, l'étude de PJM et National Grid en termes de complémentarités institutionnelles a montré que les signaux de localisation et l'internalisation des externalités d'usage du réseau électrique n'étaient pas les éléments centraux dans la coordination entre la production et le transport. Certes l'internalisation des externalités d'usage du réseau est nécessaire pour coordonner efficacement la production et le transport d'électricité à court terme et à long terme. Mais, dès lors que la localisation des producteurs est contrainte et génère à terme des congestions durables sur le réseau, les signaux de localisation sont insuffisants pour coordonner à long terme production et transport d'électricité. Seul le développement de la capacité de transport apporte une coordination satisfaisante de ces deux activités.

\footnotetext{
${ }^{9}$ Cela fonctionne aussi quand les signaux de localisation sont inopérants.
} 


\section{BIBLIOGRAPHIE}

AOKI M. [2001], Towards a comparative institutional analysis, The MIT Press, Cambridge.

BALDWIN C., CLARK K. [2000], Design Rules - The Power of Modularity. The MIT Press, Cambridge, Massachusetts et Londres, Angleterre.

BARKER J. Jr., TENEBAUM B., WOOLF F. [1997], "Governance and regulation of power pools and system operators. An international comparison", Worldbank.

BOYCE J., HALLIS A., [2005], "Governance of electricity transmission systems", Energy Economics, 27, p. 237-255.

BRUNEKREEFT G., NEUHOFF K., NEWBERY D. [2005], "Electricity transmission: an overview of the current debate", Utilities Policy, 13, p. 73-93.

JOSKOW P. [2006], "Patterns of transmission investment", dans Lévêque F. (ed.), Competitive Electricity Markets and Sustainability, Edward Elgar, p. 131-186.

LEVEQUE F. (Ed.), [2003], Transport Pricing of Electricity Network, Kluwer.

PEREZ Y. [2002], L'analyse néo-institutionnelle des réformes électriques européennes, Thèse de Doctorat en Sciences Économiques, Université de Paris I Panthéon Sorbonne.

PEREZ-ARRIAGA I. J., RUBIO F. J., PUERTA J. F., ARCELUZ J., MARIN J. [1995], "Marginal Pricing of Transmission Services: an analysis of cost recovery", IEEE Trans. on Power Systems, 10, p. 546-553.

PJM [2004]. "PJM FERC Filing in Docket Number RT-01-2-01". Avril, www.pjm.com

RIOUS .V, GLACHANT JM., PEREZ Y., DESSANTE P. [2008], “The diversity of design of TSOs”, Energy Policy 36, p. 3323- 3332.

SAUSSIER S., YVRANDE-BILLON A. [2007], Économie des coûts de transaction, Éditions La découverte, Collection Repères, Paris.

SCHWEPPE F., CARAMANIS M., TABORS R., BOHN R. [1988], Spot Pricing of Electricity. Kluwer Academic.

WILSON R. [2002], “Architecture of the power markets”, Econometrica, 70, p. 1299-1344. 